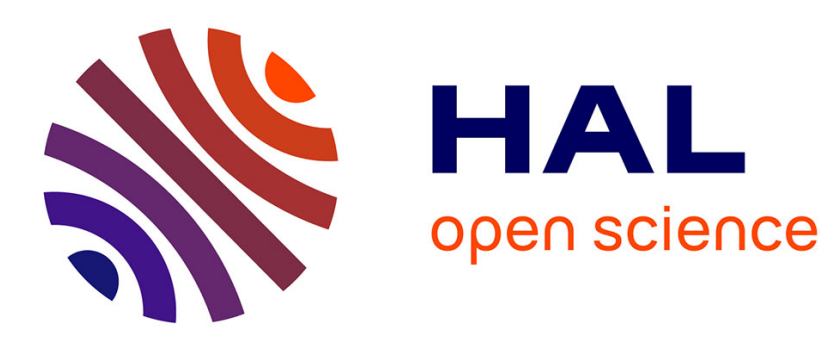

\title{
Le rapport scientifique, un observable de l'institutionnalisation de la recherche contractualisée en sciences humaines et sociales
}

Anne Piponnier

\section{- To cite this version:}

Anne Piponnier. Le rapport scientifique, un observable de l'institutionnalisation de la recherche contractualisée en sciences humaines et sociales. Mots: les langages du politique, 2017, 114, pp.135144. 10.4000/mots.22833 . hal-01718801

\section{HAL Id: hal-01718801 \\ https://hal.univ-lorraine.fr/hal-01718801}

Submitted on 27 Feb 2018

HAL is a multi-disciplinary open access archive for the deposit and dissemination of scientific research documents, whether they are published or not. The documents may come from teaching and research institutions in France or abroad, or from public or private research centers.
L'archive ouverte pluridisciplinaire HAL, est destinée au dépôt et à la diffusion de documents scientifiques de niveau recherche, publiés ou non, émanant des établissements d'enseignement et de recherche français ou étrangers, des laboratoires publics ou privés. 
Mots. Les langages du politique

$114 \mid 2017$

Le rapport, entre description et recommandation

\title{
Le rapport scientifique, un observable de
} l'institutionnalisation de la recherche contractualisée en sciences humaines et sociales

Research report and contract-based research in social sciences in France El informe de investigación y investigación bajo contrato en el ámbito de las ciencias humanas y sociales en Francia

revues.org

Édition électronique

URL : http://mots.revues.org/22833

DOI : $10.4000 /$ mots. 22833

ISSN : 1960-6001

\author{
Éditeur \\ ENS Éditions \\ Édition imprimée \\ Date de publication : 10 juillet 2017 \\ Pagination : 135-144 \\ ISSN : 0243-6450
}

\section{Distribution électronique Cairn \\ CAIRN}

CHERCHER, REPÉRER, AVANCER,

Référence électronique

Anne Piponnier, « Le rapport scientifique, un observable de l'institutionnalisation de la recherche contractualisée en sciences humaines et sociales », Mots. Les langages du politique [En ligne], 114 | 2017, mis en ligne le 10 juillet 2019, consulté le 24 juillet 2017. URL : http:// mots.revues.org/22833; DOI : 10.4000/mots.22833 


\section{Le rapport scientifique, un observable de l'institutionnalisation de la recherche contractualisée en sciences humaines et sociales}

Dans le contexte du pilotage de la recherche sur projets (Aust, 2014) ${ }^{1}$ et de l'intégration dans les politiques de recherche nationales des objectifs de compétitivité issus de l'agenda de Bologne (Bruno, 2008), des effets de cadrages d'ordre économique et institutionnel sur l'activité de recherche (Wilkas, 2009; Musselin, 2014; Piponnier, 2015) ont pu être observés. En sciences humaines et sociales, la recherche est soumise à de nouvelles normes de production (Trouche et Courbières, 2015) et d'évaluation (Bouchard, 2008). Elle inclut désormais les différents rapports de recherche ou d'expertise, issus de contrats sur financements publics ou privés. Dès lors se pose la question de leur statut et de leur légitimité scientifique dans ce nouvel environnement de travail.

Le rapport, qui bénéficie d'une certaine autonomie dans la sphère politique et professionnelle, occupe, dans la sphère scientifique, une place stratégique, celle de rendre compte de la recherche en train de se faire, d'en identifier les avancées et les résultats. Il ne peut être dissocié, dans l'espace scientifique, du régime de production et de régulation du savoir (Pestre, 2006) dans lequel il évolue et dont il est à la fois le vecteur et le reflet.

Document produit dans le cadre d'une activité de recherche contractualisée, un rapport est porteur d'informations scientifiques mais aussi sociétales et professionnelles. II relève d'un processus de travail individuel ou collectif qui s'inscrit généralement dans un dispositif de projet personnel ou d'équipe, ce dernier étant à son tour enchâssé dans un dispositif d'évaluation, lequel en retour, l'authentifie comme produit de l'activité. Le rapport, dans son unicité, ne peut donc être saisi en dehors des chaînes d'interdépendance (Elias, 1991) qui lui permettent de prendre place dans la production scientifique. C'est donc à partir de l'étude de cette configuration que nous questionnons le statut du rapport dans le champ des sciences humaines et sociales (SHS) et le processus

1. Pilotage conduit en France par l'Agence nationale de la recherche (ANR) créée en 2005 mais porté à l'échelon régional, national ou international, par diverses instances (régions, Union européenne) et des programmes cadres tels que Horizon 2020 (2014-2020), dispositif de pilotage regroupant divers programmes de recherche sur projets.

Université de Lorraine, Centre de recherche sur les médiations anne.piponnier@univ-lorraine.fr 
de légitimation dont il fait l'objet. Il s'agit là d'une question de recherche qui n'a pas encore fait l'objet d'enquêtes systématiques.

L'objectif n'est cependant pas ici de traiter de manière exhaustive d'un sujet qui reste en grande partie à défricher mais de dégager, à partir d'une étude empirique, quelques pistes de recherche pour examiner le statut de ces objets, porteurs dans l'espace académique de nouvelles formes de négociation entre recherches en sciences sociales et espace public.

Le terrain choisi pour cette étude est une équipe de recherche multidisciplinaire en SHS, à un moment stratégique de son activité, la préparation de son bilan quadriennal. Menée au cours des six mois précédant la remise du bilan, l'observation met en relief les variations d'attention vis-à-vis de ces produits de recherche mais également le travail de négociation dont ils sont l'objet à l'approche d'une échéance d'évaluation. Notre étude s'appuie sur un corpus constitué d'un ensemble de Curriculum vitaæ (CV) de chercheurs et chercheuses et de fiches d'activité individuelles (plus d'une centaine) et du recueil sur la même période des échanges par courriel entre les membres d'une équipe de l'unité à l'approche du bilan (une cinquantaine de messages). Le dépouillement des fiches d'activités permet d'observer le travail de catégorisation effectué à la source par les chercheurs. L'analyse des échanges, quant à elle, permet de suivre les négociations qui s'opèrent autour de ces catégorisations. Confronter les traces d'activité documentaire aux prises de parole que suscitent les rapports permet ainsi d'approcher au plus près les processus de qualification à l'œuvre en vue de la production du bilan collectif.

Le rapport de recherche est donc pris ici comme observable du discours scientifique, en ce qu'il figure un produit de la recherche dont on peut suivre la «carrière », depuis les mentions relevées dans les productions personnelles des chercheurs à celles figurant dans un document finalisé en commun.

Le rapport dans le champ scientifique ayant été peu exploré2, il est nécessaire en premier lieu de répertorier les types de rapports actuellement produits en SHS et de mettre en évidence la trame discursive commune qui s'est forgée dans le double contexte de la politique contractuelle et du système d'évaluation de la recherche. Ce travail liminaire étant fait, nous exposerons les résultats de notre enquête sur l'institutionnalisation des rapports au prisme de deux hypothèses : le rapport préinstitué comme un allant de soi de la production scientifique, ce que contredisent en partie les échanges; le rapport instituant un objet plastique qui se reconfigure au gré des besoins de publicisation. Notre conclusion porte sur la nécessité d'interroger le rapport comme objet de médiation des opérations de jugement portées par les acteurs sur leurs pratiques scientifiques et professionnelles.

2. Hormis l'étude de Catherine Dardy, Dominique Ducard et Dominique Maingueneau (2002) sur le rapport de soutenance de thèse. 


\section{Le rapport : un objet pluriel, une trame discursive commune}

À la question «que signifie rapporter une recherche, en particulier dans le champ des SHS? », à première vue, la réponse paraît claire : le rapport a pour finalité de rendre compte (à un commanditaire qui finance, à une instance évaluatrice qui valide) d'un projet ou d'une activité de recherche. Ce travail de restitution adressé à des destinataires identifiés indique clairement la nature de l'échange : il s'agit de donner en retour des moyens alloués, le bien symbolique que constituent les résultats de recherche, en termes de connaissances et de méthodes. Le rapport vient donc attester cet échange et informer sur la nature du contrat passé entre les acteurs. C'est un objet de communication qui actualise le dialogue entre les parties prenantes tout en en conservant la mémoire.

\section{Un objet de la culture du contrat}

À l'instar de l'espace professionnel et institutionnel où le rapport constitue un outil de pilotage de l'activité, dans l'espace de la recherche les rapports peuvent varier selon le type de contrat et d'usage - rapport intermédiaire de recherche, rapport d'étape du bilan d'une unité de recherche, rapport d'étude ou d'expertise, rapport d'évaluation, rapport de projet financé. Ils sont la marque d'un contrat (institutionnel, financier, moral) passé entre les chercheurs et leurs partenaires et de l'émergence d'une culture de la recherche contractualisée en SHS.

Cette culture naissante est sous-tendue par la figure du projet, un idéaltype de la régulation de l'activité par le contrat, en ce qu'il définit et concrétise les termes du dialogue entre chercheurs, institutions, experts et société civile. Le pilotage par projets incarne précisément ce mode de gouvernance. Largement répandu dans tous les domaines de l'action publique, il est considéré par les institutions comme une réponse adaptée aux impératifs de compétitivité et d'innovation (Lascoumes et Le Galès, 2004). En s'appuyant sur une complémentarité de compétences et une dynamique collective, il est censé garantir une voie démocratique et établir un dialogue de gestion entre les parties (tutelles administratives, partenaires scientifiques, économiques et sociaux), pour résoudre un certain nombre de problèmes publics.

Le rapport est ainsi à la fois un document contractuel entre les parties prenantes mais aussi un texte, au sens premier du terme, puisqu'il tisse étroitement de multiples registres de langue et d'écriture d'ordre scientifique, technique et administratif. Les rapports sont donc pluriels tant par leurs modes d'énonciation que par leurs conditions d'usage. Néanmoins, ils ont en commun une trame discursive liée au dialogue contractuel, à travers l'usage de règles d'écriture qui permettent à ce dialogue de prendre forme. 


\section{L’autorité médiatrice du formulaire}

Certains rapports de recherche en SHS ne sont pas directement soumis à des règles d'écriture autres que celles en vigueur dans la rédaction scientifique telles que les rapports adressés entre pairs dans la phase de préparation d'un projet par exemple. Mais une très grande majorité de rapports s'appuient sur des modèles existants plus ou moins incorporés, issus de diverses traditions administrative, organisationnelle et comptable, ce qui est notamment le cas pour la rédaction aussi bien d'un bilan annuel d'activité que d'un rapport de projet financé. En nombre croissant dans l'activité de production de recherche, et, en particulier dans la communauté SHS observée, les rapports rédigés dans la cadre de programmes ou projets de recherche financés sont élaborés sur la base d'un formulaire servant de trame pour la rédaction du rapport.

La médiation du formulaire défini par Roy Harris (1993), comme « une forme d'après laquelle les actes doivent être rédigés», est ici décisive pour analyser la construction des énoncés à la croisée des différents registres. Les formulaires sont des matrices proposées pour la rédaction d'un rapport et varient peu d'un projet à un autre3. Ils reprennent généralement presque à l'identique les éléments du formulaire utilisé pour la soumission du projet auprès de l'agence (Piponnier, 2012). Structurés en une dizaine de rubriques (hors annexes) à renseigner, ils donnent pour chacune d'entre elles, des consignes de rédaction telles que le caractère obligatoire des renseignements, le nombre de signes requis, l'insertion d'éléments illustratifs ou explicatifs des tableaux et figures, etc.

Exemple à titre illustratif de modèle de fiche.

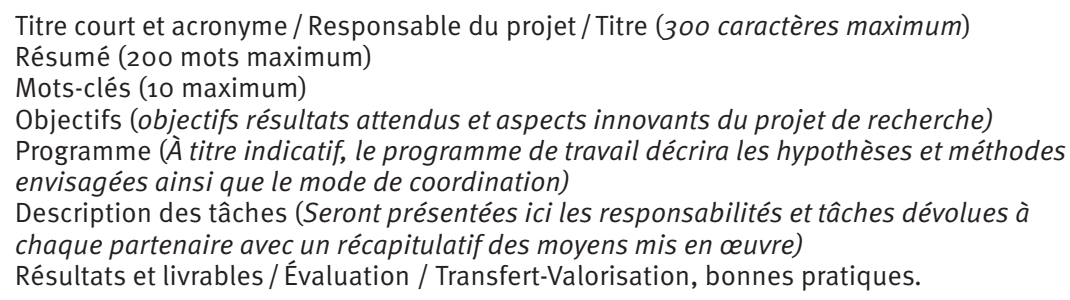

Source : France, ministère de la Culture et de la Communication, 2016

3. Ni spatialement d'un appel à l'autre, ni au cours du temps : il est intéressant de noter ici la relative stabilité du formulaire alors même que les programmes se diversifient en nombre et en genre, et que les systèmes d'appels se sont largement densifiés et complexifiés depuis nos premières observations effectuées il y a plus d'une dizaine d'années. 
Si le formulaire sert d'embrayeur à la rédaction tout en cadrant son contenu, il constitue simultanément une ressource pour les évaluateurs. À travers ses recommandations, il agence deux registres de discours, l'un de nature gestionnaire (détailler l'organisation matérielle du projet), l'autre de nature scientifique (exposer les hypothèses de travail, justifier les méthodologies et modes opérationnels) et contribue à leur hybridation : les indications données pour définir les objectifs, dégager les résultats ou encore défendre les stratégies de valorisation et de communication, tissent ensemble un interdiscours, propre aux rapports de recherche financée.

Cette matrice répliquée dans de très nombreux cadres d'appel en vigueur (par exemple dossiers de soumission, guides pratiques pour la rédaction des rapports, fac-similé de formulaires), et que les documents d'expertise et d'évaluation de l'activité réutilisent à leur tour, tend à construire un modèle de référence, sur lequel les rédacteurs s'appuient pour bâtir leurs rapports. Ce modèle qui circule largement n'est pas sans incidence sur la connaissance tacite qui se construit autour du rapport dans l'espace de la recherche et sur la place que les chercheurs lui réservent dans le recensement de leurs productions.

\section{La place du rapport dans l'activité du chercheur : parcours d'une reconnaissance}

La place attribuée au rapport dans une production de recherche dépend ainsi étroitement de la culture du contrat de l'équipe, de l'importance qu'elle accorde à ce mode de recherche et des médiations - techniques, administratives, scientifiques - qu'elle met en place pour le développer. Dans la communauté observée, la culture du contrat est relativement bien installée, comme en témoignent le nombre et la diversité des contrats obtenus ou en cours4.

Regardons néanmoins de plus près comment l'inscription du rapport s'effectue dans les documents produits par l'équipe pour se constituer comme produit de la recherche.

\section{Un marqueur instable de l'activité}

Une première étape de notre enquête avait pour but de repérer via les CV le travail d'identification des rapports dans l'activité de recherche. La publication du CV sur l'espace collaboratif de travail de l'unité n'a pas de caractère obligatoire et, lorsqu'il est rendu disponible, sa rédaction reste libre. Néanmoins les

4. Cet article n'ayant pas vocation à l'évaluation, nous ne livrons pas de chiffres précis. Notons toutefois que dans cette unité de recherche interdisciplinaire, le pourcentage de contrats est supérieur à la moyenne observée dans les unités de composition et de taille équivalentes. Source : Haut Comité de l'évaluation de la Recherche et de l'Enseignement supérieur (HCERES). 
CV sont généralement structurés selon un modèle générique en usage, héritier des différentes politiques et procédures d'évaluation. L'activité y est répartie en trois volets distincts 5 : éléments biographiques, activités d'enseignement et activités de recherche. Les rapports figurent dans ce dernier volet, de manière diverse, parfois redondante : à la fois dans la description des activités de recherche conduites en propre ou en collaboration, et dans la liste des publications du chercheur. Ces mentions multiples montrent que si le rapport est bien présent dans la production de recherche, il s'énonce comme objet dans l'action (Akrich, 1993), c'est-à-dire comme support de l'activité de recherche et produit de cette activité. Le rapport est ainsi d'abord un marqueur de l'activité. La mention effective des rapports dans l'ensemble des volets recherche des CV semble donc dessiner une forme de consensus implicite sur la pertinence à valoriser les produits issus de recherches contractualisées.

Les CV sont classiquement accompagnés d'une liste de publications permettant de détailler et catégoriser la production. L'élaboration de cette liste s'appuie sur les recommandations du HCERES qui définit une typologie des produits de recherche, parmi lesquels les rapports constituent une catégorie à part entière. Cette liste dite de "publications » est sujette à diverses interprétations : le terme réfère à une typologie restrictive - documents soumis à l'examen d'un travail de révision par les pairs - que le détail des catégories proposées vient élargir en incluant diverses productions qui ne sont pas (nécessairement) soumises à ce processus tels que les rapports, les productions créatives théorisées, les productions de la communication scientifique et technique par exemple. Face à cette incertitude, des attitudes contrastées ont pu être observées, que l'on ne peut pas directement corréler à l'expérience du chercheur dans la conduite de recherches contractualisées : lorsque celleci est forte - conduite d'au moins un contrat annuel -, le rapport est indiqué dans la catégorie appropriée (rapports) mais ne fait pas nécessairement l'objet d'un signalement précis. Lorsque l'activité est naissante ou ponctuelle, plusieurs cas de figure sont observés : une absence de mention d'un rapport - cas à la marge -, un signalement approximatif - intitulé incomplet, pas de mention des commanditaires - ou encore, à l'inverse, un signalement exhaustif du type :

〈Auteur(s), Date, Titre, Type de rapport, Lieu, Institutions concernées, nombre de pages>

À l’instar des documents non destinés à être publiés - archives, notes, journaux de terrain par exemple -, le rapport n'est que peu disponible à la commu-

5. Ce modèle est issu des recommandations faites par les instances de pilotage et d'évaluation telles que l'Agence d'évaluation de la Recherche et de l'Enseignement supérieur (AERES) devenu le HCERES en 2013 et par les sections du Conseil national des universités. Il est également largement utilisé dans les dossiers des candidats aux fonctions d'enseignant-chercheur. 
nauté, si ce n'est dans le bureau du chercheur et souffre d'un déficit de signalement propre à ce type de document ${ }^{6}$. D'abord destiné à un usage restreint, le rapport appartient à ce qu'il est convenu d'appeler la littérature grise : il est d'abord adressé aux parties prenantes, même si dans l'espace académique, il peut circuler auprès des chercheurs, équipes-projet, unités de recherche, instances d'évaluation. Ce statut documentaire peut expliquer en partie l'imparfaite publicisation des rapports7. Mais bien que peu ou mal référencé dans les CV et certaines publications, le rapport semble jouer de sa performativité : signaler un rapport, même de manière incomplète, indique qu'une recherche contractualisée a bien eu lieu et suffit à attester la production d'un savoir afférent. Ainsi, malgré une qualification relativement instable, le rapport apparaît comme une production de recherche.

\section{De la qualification à la reconnaissance}

Dans l'unité observée, les chercheurs ont été invités à produire des fiches individuelles de recherche sur la base d'un formulaire-type ${ }^{8}$. Destinées à un usage interne - recenser les profils et les productions de recherche en vue de la préparation du bilan -, ces fiches fournissent des renseignements intéressants sur le processus d'incorporation de normes et leur acceptabilité auprès des chercheurs. Une observation des échanges nés à l'occasion de la rédaction de ces fiches et de leur collecte au sein de l'unité nous montre que le rapport est un objet qui ne va pas nécessairement de soi et qu'il est particulièrement exposé au travail d'ajustement et d'articulation (Strauss, 1988) à l'œuvre dans l'activité de rationalisation collective que constitue la préparation d'un bilan.

L'accueil de la demande de fiches individuelles a été mitigé, nombre de chercheurs acceptant mal un travail administratif supplémentaire dans un contexte professionnel déjà très alourdi par ces tâches, entre réponses aux appels à projet, montages de dossiers de financement et recherches de partenariats. Par ailleurs le retour de cette fiche individuelle, même à usage strictement interne, n’a pas toujours été bien reçu par certains chercheurs voyant là ressurgir un outil de contrôle de l'activité et d'évaluation indirecte. Mais au final l'ensemble de l'unité s'est plié à l'exercice, acceptant bon gré mal gré l'intérêt supérieur de l'unité.

6. En effet, les recommandations faites aux auteurs pour la rédaction de leurs références bibliographiques ne mentionnent généralement pas le rapport comme exemple-type alors même qu'il est identifié en tant que tel dans les différentes normes en vigueur (notamment les normes APA ou encore ISO 690).

7. Tout au moins, ceux qui n'ont pas fait l'objet d'une publication chez un éditeur commercial ou institutionnel.

8. Formulaire adapté en interne de la fiche individuelle proposée initialement par l'AERES puis supprimée dans le cadre de la loi relative à l'Enseignement supérieur et la Recherche du 22 juillet 2013 et portant création du HCERES. 
Le recueil des fiches a donc nécessité un certain nombre de médiations pour convaincre de leur intérêt et expliquer les modalités de leur rédaction. A priori compléter ces fiches et les mettre à jour avec les publications les plus récentes ne devait pas constituer un gros problème, les fiches s'appuyant sur la structure fournie par le HCERES et dont se servent les chercheurs pour le classement de leurs publications dans leurs CV. Mais le transfert des informations du CV à la fiche a soulevé diverses questions et a fait l'objet de réglages qui ont révélé à la fois des représentations assez diverses de l'activité et des perceptions assez contrastées de l'intérêt de signaler certaines productions. Les négociations ont porté sur trois plans : l'identification du rapport comme objet autonome, son signalement, le statut de l'auteur lorsqu'il s'agit d'une production collective.

Le premier constat est que le rapport est un objet qui peine à s'autonomiser : corrélé à une activité de restitution de la recherche, le rapport est inclus dans l'énoncé de cette activité. Détacher le rapport de ces conditions de production, pour le signaler comme produit de la recherche, n'est pas considéré comme un problème en soi mais pose à certains acteurs la question d'éventuels effets de doublons dans la fiche, un point jugé critique au regard des procédures d'évaluation. Pour être recensés sans risque de répétition mal interprétée, les rapports doivent donc pouvoir être identifiés sans ambiguïté. Si l'insertion dans la catégorie Rapports ne fait pas débat, leur référencement suscite commentaires et demandes de précisions pour la mise aux normes bibliographiques. Une médiation se met alors en place dans les échanges pour faire circuler les savoirs et les bonnes pratiques et trouver un consensus sur l'interprétation de la norme et un usage approprié dans le contexte d'évaluation. Un dernier point de discussion concerne la question de l'attribution (Pontille, 2004) du rapport à un chercheur en particulier, lorsque le rapport est signé collectivement et plus encore lorsque le chercheur n'en a été qu'un des collaborateurs. Les chercheurs concernés interrogent la pertinence de signaler un rapport dont ils ne se jugent pas le seul auteur et de s'en attribuer ainsi la responsabilité scientifique. Au-delà de la question de l'honnêteté intellectuelle, se profile dans les prises de parole la question pendante du chercheur-auteur dans un contexte d'activité qui repose de plus en plus sur le collectif et une production discursive polyphonique. Cette question actualise dans le domaine de la publicisation de la recherche, la question de la distinction entre auteur et image d'auteur (Maingueneau, 2009) et ouvre, en particulier dans le contexte de la recherche contractualisée, de nouvelles pistes d'analyse.

En observant le processus de reconnaissance des rapports au sein d'une équipe de recherche, notre étude vient interroger l'habitus scientifique d'une communauté de recherche en SHS confrontée à de nouvelles formes de rationalisation de son activité liées aux conditions de production de la recherche dans la nouvelle économie. La carrière des rapports dans la production individuelle et collective de l'équipe, qui résulte de multiples négociations, témoigne, 
de ce point de vue, de la disposition des chercheurs à s'emparer d'injonctions technico-administratives pour requalifier une production qui interroge la nature et l'enjeu de leur rapport à leur métier de chercheur.

\section{Conclusion}

L'arrivée en force du rapport dans le champ de l'activité professionnelle n'est pas propre au secteur de la recherche. Dans ce domaine comme dans ceux de la santé, de l'environnement, de l'éducation ou encore de la culture, le rapport est un maillon stratégique d'un dispositif administratif et politique qui fait reposer sur un certain nombre d'instruments (Lascoumes et Le Galès, 2004) la charge de piloter l'action publique.

Dans le domaine de la recherche, l'action conjuguée du développement des contrats et des nouvelles règles d'évaluation de la recherche contribuent à modifier la place et le statut du rapport. À travers les différentes façons de le nommer, l'enregistrer, le qualifier dans son environnement professionnel, le chercheur fait du rapport une production de savoirs à part entière. Ancrée dans un double procès de production et de publicisation à l'épreuve de dispositifs reposant sur une articulation étroite entre des objectifs d'évaluation, d'accountability et de transparence dont elle est chargée d'assurer la légitimité et l'acceptabilité, la qualification des rapports dans le champ scientifique nous informe sur le processus de réinstitutionnalisation de la recherche à la croisée de plusieurs mondes (académique, expert, politique, citoyen).

\section{Références}

AKRICH Madeleine, 1993, «Les objets techniques et leurs utilisateurs, de la conception à l'action », Les objets dans l'action, B. Conein, N., Dodier et L. Thévenot éd., Paris, Éd. de l'EHESS, p. 35-57 ("Raisons pratiques», nº 4).

AUsT Jérôme, 2014, "Financer la recherche sur projet. Figures historiques d'un dispositif de gouvernement », Genèses, nº 94, p. 2-6.

BOUCHARD Julie éd., 2008, "La communication nombre», MEl, Médiation et information : revue internationale de communication, $n^{0} 28$, p. 1-4.

BRUno Isabelle, 2008, À vos marques, prêts... Cherchez! La stratégie européenne de Lisbonne, vers un marché de la recherche, Bellecombes-en-Bauges, Éd. du Croquant.

DARDY Claudine, DuCARD Dominique, MaIngueneau Dominique, 2002, Un genre universitaire. Le rapport de soutenance de thèse, Villeneuve-d'Ascq, Presses universitaires du Septentrion.

ELIAS Norbert, 1991 [1981], Qu'est-ce que la sociologie?, La Tour-d'Aigues, Éd de l'Aube. FontANIER Pierre, 1968, Les figures du discours, Paris, Flammarion. 
Hagstrom Warren O., 1965, The Scientific Community, New York, Basic Books.

HARRIS Roy, 1993, Sémiologie de l'écriture, Paris, Éd. du CNRS.

LASCOUmes Pierre, Le GALÈs Patrick éd., 2004, Gouverner par les instruments, Paris, Presses de Sciences Po.

Latour Bruno, Woolgar Steve, 1988 [1979], La vie de laboratoire, Paris, La Découverte.

MAINGUENEAU Dominique, 2009, "Auteur et image d'auteur en analyse du discours», Argumentation et analyse du discours, $\mathrm{n}^{\circ} 3$, disponible sur Internet 〈http://aad. revues.org/660> (consulté le 13 mars 2017).

MusSELIN Christine, 2014, "Les effets paradoxaux du développement des financements sur projets et de l'évaluation sur les universités et la profession universitaire», Communication au Séminaire Questionner l'évaluation dans l'enseignement supérieur et la recherche, organisé par l'Institut des Sciences et de la Communication, jeudi 6 mars, Paris, CNRS.

Pestre Dominique, 2006, Introduction aux «Science Studies », Paris, La Découverte.

PIPONNIER Anne, 2010, «Publier la recherche en mode projet : objets et pratiques entre innovation et normalisation », Actes du colloque international Edition et publication scientifiques en sciences humaines et sociales, formes et enjeux, Y. Jeanneret éd., Avignon, Université d'Avignon et des Pays-du-Vaucluse, p.193-203.

PIPONNIER Anne, 2012, "L'espace sémiotique du projet : du dispositif médiatique au territoire symbolique», Communication au colloque Communication du symbolique et symbolique de la communication dans les sociétés modernes et postmodernes, Université Paul-Valéry-Montpellier 3, 8 et 9 novembre 2012.

PIPONNIER Anne, 2015, "Le projet dans les pratiques de recherche. Pour un retour critique sur nos engagements », Sciences de la société, nº 93, p.111-123.

PONTILLE David, 2004, «Signature et travail d'attribution. Les ressorts de l'auteur scientifique », Réseaux: communication, technologie, société, nº127-128, p. 253-275.

STRAUSS Anselm, 1988, «The Articulation of Project Work. An Organizational Process », The Sociological Quarterly, vol. 29, nº 2, p.163-178.

Trouche Dominique, Courbieres Caroline, éd., 2015, "La recherche sur projet en sciences humaines et sociales : lieux, stratégies et contenus», Sciences de la société, nº 93, p.3-11.

VILKAS Catherine, 2009, «Des pairs aux experts : l'émergence d'un nouveau management de la recherche scientifique? », Cahiers internationaux de sociologie, n ${ }^{0} 126$, p. 61-79. 